\title{
AN ONTOLOGY FOR A WEB DICTIONARY OF ITALIAN SIGN LANGUAGE
}

\author{
Rosella Gennari \\ KRDB, Free University of Bozen-Bolzano, Piazza Domenicani 3, 39100 Bolzano \\ gennari@inf.unibz.it \\ Tania di Mascio \\ University of L'Aquila, I-67040 Monteluco di Roio, L'Aquila \\ tania@ing.univaq.it
}

Keywords: ontology and the semantic web; knowledge management; web-based education.

\begin{abstract}
Sign languages are visual languages used in deaf communities. They are essentially tempo-spatial languages: signs are made of manual components, e.g., the hand movements, and non-manual components, e.g., facial expressions. The e-LIS project aims at the creation of the first web bidirectional dictionary for Italian sign language-verbal Italian. Whereas the lexicographic order is a standard and 'natural' way of ordering hence retrieving words in Italian dictionaries, there is nothing similar for Italian sign language dictionaries. Stokoebased notations have been successfully employed for decomposing and ordering signs in paper dictionaries for Italian sign language; but consulting the dictionaries requires knowing the adopted Stokoe-based notation, which is not as easy-to-remember and well-known as Italian alphabet is. Users of a web dictionary cannot be expected to be expert of this. There the role of ontologies comes into play. The ontology presented in this paper analyses and relates the formational components of a sign; in some sense, the ontology allows us to 'enrich' the e-LIS dictionary with expert information concerning classes of sign components and, above all, their mutual relations. We conclude this paper with several open questions at the intersection of knowledge representation and reasoning, semantic web, sign and computational linguistics.
\end{abstract}

\section{INTRODUCTION}

Sign languages are visual languages used in deaf communities, mainly. They are essentially tempospatial languages, simultaneously combining shapes, orientations and movements of the hands, as well as non-manual components, e.g., facial expressions. A sign language and the verbal language of the country of origin are generally different languages. The creation of an electronic dictionary for Italian sign language is part of the e-LIS project (E-LIS project, 2004), which is lead by the European Academy of Bozen-Bolzano. The project commenced at the end of 2004 with the involvement of the ALBA cooperative from Turin, active in deaf studies. Section 2 outlines the essential background on Italian sign language and the e-LIS project's history.

Initially, the e-LIS dictionary from Italian sign language to verbal Italian was intended for expert signers searching for the translation of an Italian sign. At the start of 2006, when the development of e-LIS was already in progress, it was realised that potential users of a web dictionary would also be non-experts of Italian sign language. Then the idea of an ontology and the associated technology for the dictionary took shape. We explain our ontology in Section 3 , and comment on it in Section 4 Section 5 outlines the architecture of the ontology-driven dictionary, and the role that our ontology plays in it. In Section 6 , we compare our ontology-driven dictionary to other electronic dictionaries for sign languages. Section 7 concludes this paper with an assessment of our work and several open questions.

\section{BACKGROUND}

\subsection{Sign language and dictionaries}

A sign language (SL) is a visual language based on body gestures instead of sound to convey meaning. SLs are commonly developed in deaf communities. 
They can be used to discuss any topic, from the simple and concrete to the lofty and abstract.

Contrary to popular belief, SL is not universal; SLs vary from nation to nation; even more, SLs such as Italian sign language (LIS) have dialects of their own. LIS is not a visual rendition of Italian verbal language; LIS has a grammar, syntax and lexicon of its own, e.g., a word can be translated into more than one sign and vice versa (see Figure 1).

As highlighted in (Pizzuto et al., 2006), SLs can be assimilated to verbal languages "with an oral-only tradition"; their tempo-spatial nature, essentially 4dimensional, have made it difficult to develop a written form for them. "However" - as stated in (Pizzuto et al., 2006) - "Stokoe-based notations can be successfully employed primarily for notating single, decontextualized signs' 17 As such, they are used to transcribe signs and order them in (Radutzky, 2001), a paper dictionary of LIS to Italian.

The transcription is based on a decomposition of signs into so-called 'formational units'. The following classes correspond to the formational units adopted in (Radutzky, 2001):

- the handshape class collects the shapes the hand/hands takes/take while signing; this class alone counts more than 50 terms in LIS;

- the orientation class gives the the palm orientations, e.g., palm up;

- the movement of the hand/hands class lists the movements of the hands in LIS;

- the location of the hand/hands class provides the articulation places, i.e., the positions of the hands (e.g., on your forehead, in the air).

Let us see an example entry of (Radutzky, 2001): the sign for "parlare dietro le spalle" (to gossip behind one's back) in Figure 1 is a one-hand sign; the handshape is flat with five stretched fingers; as for the orientation, the palm orientation is forward and towards the left so that the hand fingers get in touch once with the location which is the neck; as for the movement of the hand, this moves to the left only once.

This information is readable in the transcription in the upper-left corner of Figure 1 (namely, $\mathbf{B}_{\perp<} \Pi$ *•). However, figuring out this information from the transcription requires some expert knowledge of the adopted transcription system and, above all, of the underlying formational rules of signs.

Before proceeding further, a word on the written representation of SLs is in order. The representation of SLs in written form is a difficult issue and a topic

\footnotetext{
${ }^{1}$ They were adopted for transcribing and ordering American signs in (Stoke et al., 1965).
}



Figure 1: Sign for Italian expression Parlare dietro le spalle, as in (Radutzky, 2001).

of current research, e.g., see (Garcia, 2006, Pizzuto et al., 2006). We do not discuss this here for it goes beyond the scopes of our work which, at present, is of experimental nature mainly.

\subsection{The e-LIS project}

The e-LIS dictionary is part of a research project lead by the European Academy of Bozen-Bolzano (EURAC); e-LIS stands for dizionario Elettronico per la Lingua Italiana dei Segni (Electronic dictionary for LIS).

The project was conceived at the end of 2004 at EURAC (E-LIS project, 2004). The ALBA cooperative from Turin, active in deaf studies, was involved in the project for the necessary support and feedback on LIS. As clearly stated in (Vettori et al., 2004), most sign language dictionaries form a hybrid between a reference dictionary and a learner's dictionary. On the contrary, the e-LIS dictionary is conceived a 'semibidirectional dictionary', explaining LIS signs using LIS as meta-language and vice-versa.

The electronic format is particularly suited to an SL; for instance, it allows for videos and animations to be integrated in the dictionary and used to render the movement of signs in space. Since the dictionary aims at reaching as many users as possible, it was conceived as a web application.

However the dictionary from LIS to verbal Italian was initially intended only for expert signers searching for the translation of a sign into verbal Italian. Only subsequently it was realised that the potential users of a web dictionary could also be non-experts of LIS, willing to learn it; we cannot expect that they become expert of the transcription system outlined in Subsection 2.1 and, in particular, that they know how to compose the formational units of signs of that system. Then, at the start of 2006 , we commenced to 
work on a domain ontology (Guarino, 1998) for the LIS-to-Italian dictionary of e-LIS in order to represent and make available to all such a knowledge.

In the following Sections 3 and 4 , we focus on the domain ontology at the core of our ontology-driven dictionary, whose architecture is outlined in Section 5 .

\section{THE DOMAIN ONTOLOGY}

The domain of our e-LIS ontology is the Stokoe-based classification outlined in Subsection 2.1 above. The ontology was constructed in a top-down manner starting from (Radutzky, 2001) with the expert assistance of linguists and deaf users of the e-LIS project (see Subsection 2.2. It was designed using the ICOM ontology editor (Fillottrani et al., 2006).

Note that our ontology is 'richer' than that classification: the ontology introduces novel classes and relations among classes, thereby making explicit relevant pieces of information which were implicit and somehow hidden in that classification and in the reference paper dictionary. For instance: it makes explicit that each one hand sign is composed of at least one handshape by introducing an appropriate relation among the corresponding classes, One-hand sign and Handhsape; it groups together all the twelve different types of cyclic movements of hands in the Movement in circle class, not present in the paper dictionary.

The current domain ontology in diagrammatic format and a snippet of it are shown in Figure 2 (see also the high-resolution version (E-LIS ontology, 2006)). In the remainder, we focus on the main classes and relations of the ontology, explaining their role and our motivations for their creation. We restrict our exposition to the essential features of the ontology. For instance, cardinality constraints are missing in our below exposition, because they can be easily understood from our ontology available online at (E-LIS ontology, 2006). However, note that they are integral part of our domain ontology.

\subsection{Composed and simple signs}

Some LIS signs are 'composed' of 'simpler' signs; for instance, a composed sign may specify two locations for the dominant hand location, that is, the initial location and the final location the hand assumes due to a certain movement. To account for this distinction in our ontology,

- the Sign class is partitioned into the Composed sign and Simple sign subclasses (see the top of the ontology snippet in Figure 2,
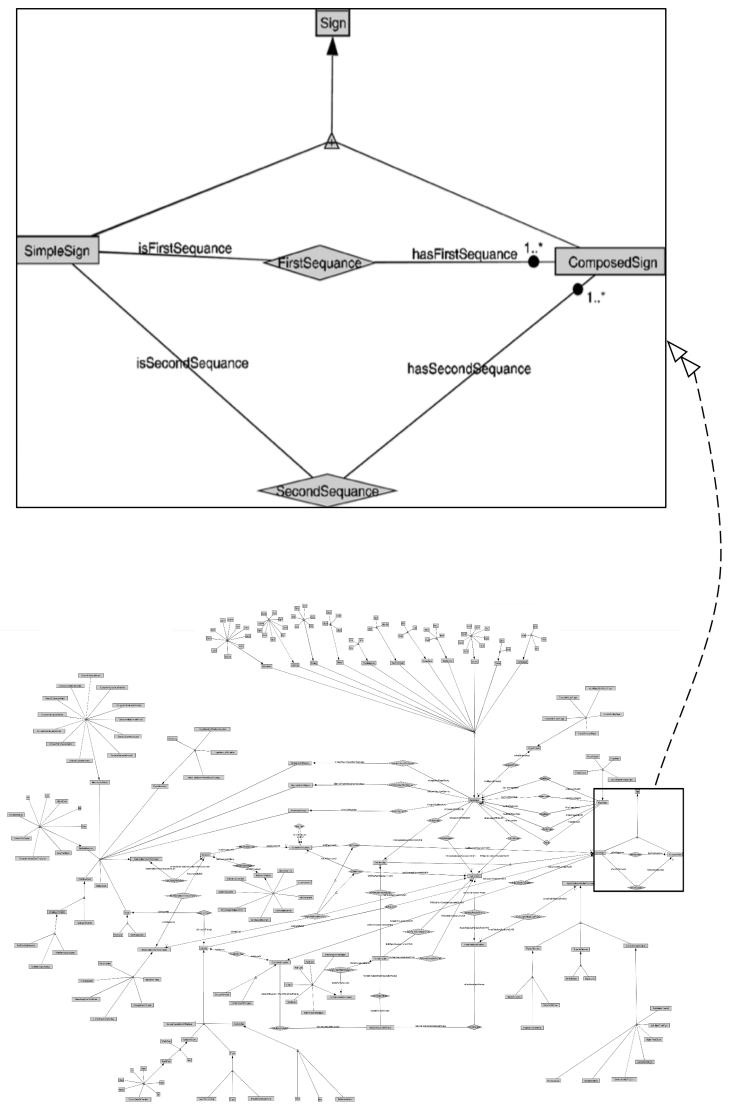

Figure 2: At the bottom: the ontology diagram. At the top: a snippet of the ontology diagram. See also the highresolution version (E-LIS ontology, 2006).

- two relations are introduced between the Simple sign and Composed sign classes, stating that each composed sign is made precisely of two simple signs (see First sequence and Second sequence in the ontology snippet in Figure 2).

The One-hand sign and Two-hand sign classes form a partition of Simple sign, meaning that each simple sign is either a one-hand sign or a two-hand sign. By inspection of (Radutzky, 2001), we abstracted the rule-based definitions of one-hand sign and two-hand sign provided in Table 1 in BNF-notation, where: $\mathrm{H}$ stands for handshape; Loc gives the hand location; $\mathrm{O}$ specifies the palm orientation; $\mathrm{C}$ stands for the contact location of the hand; $\mathrm{R}$ gives the hand relational position and occurs in two-hand signs only; MovSeq is a sequence of at most three movements (M). All can be referred to the dominant hand $(\mathrm{DH})$ or the non-dominant hand (NDH), except R that can only be referred to the non-dominant hand - the dominant hand is the right hand and the non-dominant hand is the left hand for a right-handed person. 


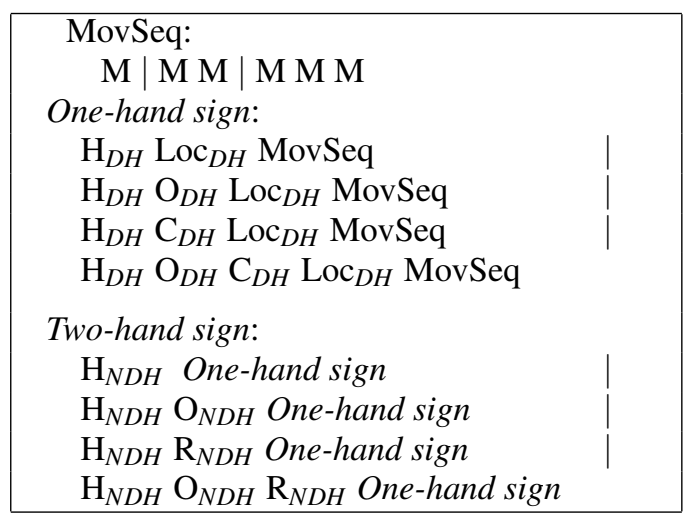

Table 1: Definitions of One-hand sign and Two-hand sign as in the e-LIS domain ontology.

For instance, according to this definition, both one-hand signs and two-hand signs specify the handshape $(\mathrm{H})$ and location (Loc) of the dominant hand $(D H)$; moreover the two-hand sign also specifies the handshape of the non-dominant hand $(N D H)$.

Our e-LIS ontology makes explicit these rules for the One-hand sign and Two-hand sign classes and the related subclasses; we explain how in the remainder of this section. In particular, whenever the dominant or the non-dominant hand relate two of these classes, we have a corresponding relation in the ontology. For instance, the ontology has two relations between Handhsape and Two-hand sign, one concerning the handshape of the dominant hand, and the other concerning the handshape of the non-dominant hand; instead, the ontology sets only one relation for the handshape of one hand (the dominant hand) between Handhsape and One-hand sign. In this manner, the implicit semantics of one-hand signs and two-hand signs is correctly represented.

\subsection{Location and contact with location}

As specified in Table 1, contact with location and location are properties of both one-hand signs and twohand signs, but they pertain to the dominant hand only (*). In our ontology, One-hand sign and Two-hand sign partition Simple sign. Then, to account for $\left(^{*}\right)$, the ontology has a relation named DHContactWithLocation between the Simple sign and Contact with location classes, and a relation named DHLocation between the Simple sign and Location classes.

Location. The Location class is subdivided into four main subclasses:

- Neutral space in front of the body;
- Arm or its part (divided in Wrist, Arm, Non dominant hand);

- Trunk (divided in Lower trunk and hip, Chest, Shoulders and upper trunk);

- Neck and above (divided in Neck, Whole face, Part of face).

In turn, the Part of face subclass of Neck and above is divided into:
- Cheek;

- Chin;

- Eye;

- Top and sides of the head;

All the above classes, with the exception of the following abstract concepts

- Arm or its part,

- Trunk,

- Neck and above,

- Part of face,
Contact with location. By inspection of (Radutzky, 2001), we can see that there are two possible kinds of location contacts: the first one is the hand contact and the other one is the finger contact. Thus in our ontology we have the Contact with location class divided into two subclasses, namely,

- Contact with hand,

- Contact only with fingers.

We have then a relation between the Contact with location class and the related Location class to express the contact of either the hands or the fingers with a body location. The ontology has also a relation between the Contact with location class and the Hand or hands initial position class to express that the contact with location has to be specified for the initial position of the dominant hand, in two hand signs or one hand signs.

\subsection{Movement}

The movement category is the most complex one. The whole movement is made of one or more sequences, which are built out of one single movement of the hand/hands (see Table 1). To account for this behaviour, the ontology has the Movement in sequence class and the Movement class, as well as a relation between them to express that the Movement in sequence class is responsible for building sequences of movements. Some components of the movement concern the dominant hand while others concern the nondominant hand (see 184.2 in (Radutzky, 2001)), but 
'which is which' is not made explicit by the classification adopted in the paper dictionary (see Table 1); thus the ontology has two types of relations, one for the dominant and the other for the non-dominant hand, and involving the Movement in sequence class.

Furthermore, the movement in sequence property is common to the One-hand sign and Two-hand sign classes; the ontology expresses this via a relation between the Simple sign class and the Movement in sequence class.

The Movement attribute class provides attributes of the Movement class, and is subdivided in:

- Elbow stretching;

- Slow movement;

- Continuous movement;

- Finger sequential movement;

- Held movement; - One time repeated;

- Stretched move- • Alternating movement. ment;

A movement is built out of two kinds of components:

- one-hand movement component;

- relational movement component.

The ontology expresses this via the related subclasses of the Movement class, explained below.

One-hand movement. The One-hand movement class is itself divided into:

- Movement in circle, e.g., convex clockwise frontal;

- Directed movement, e.g., up and down;

- Finger movement, e.g., crumbling;

- No movement;

- Touch, e.g., with hand;

- Wrist movement, e.g., twisting at the wrist.

The above classes are further partitioned as in the paper dictionary. In the ontology (and not in the paper dictionary), the Touch subclass has a relation with the Location class; this relation makes it explicit that the movement of type "touch" is related to the signer's body or the neutral space in front of the body. In the latter case the sign is a two-hand sign (see 700.1 in (Radutzky, 2001)).

Relational movement. The Relational movement class characterizes all the movements of a hand with respect to the other. It is thus divided into:

- Hand insertion;

- Hands towards each other;

- Crossing hands;

- Hands away

- Change place of hands; from each other;
The Relational movement class has a relation with the Two-hand sign class to express that the relational movement applies to two-hand signs only. To make it clear that this relation concerns both hands, we made the relation inherit from

- the relation between the Simple sign and the Movement in sequence classes, and concerning the dominant hand,

- the relation between the Two-hand sign and the Movement in sequence classes, and concerning the non-dominant hand.

\subsection{Handshape}

Now we focus on the handshape category, which is specified for the dominant hand only in one-hand signs, and for both hands in two-hand signs (see Table 1). Therefore our ontology explicitly introduces a relation between One-hand sign and Handshape, and two relations between Two-Hand sign and Handshape.

The Handshape class is divided in the eleven subclasses of handshapes listed in (Radutzky, 2001):
- Extensions;
- Opening
- Closing;
- Closed;
- Crumblinglike;
- Round shaped;

- Closed fists;

- Rectangular;

- Curved;

- Flat shaped;

- Others.

Each of the above subclasses is further subdivided as in the paper dictionary, and there is nothing new in our ontology with respected to this.

Let us turn to something new. By careful inspection of the paper dictionary, one sees that the handshape changes due to the following movements (a critical feature for the interface design of the eLIS dictionary driven by the ontology): closing hand or fingers; opening hand or fingers; configuration change. To express this information in the ontology we have three relations between the Handshape and the Closing hand or fingers, Opening hand or fingers, Configuration change classes. Moreover we have five relations for all the fingers of the hand between the Handshape and Finger state classes.

Note that the Finger state class is not present in (Radutzky, 2001). We have it explicitly in our ontology to show that the configuration depends on a given state of the fingers. It is divided into:

- Finger closed; - Finger bent;

- Finger - Finger bent at palm knuckles. straight; 
The Handshape change class is not present in the paper dictionary, however it is used in it (e.g., see 361.3). Another new class is the Finger contact class. It is divided into:

- Thumb with middle finger;

- Thumb with ring finger;

- Thumb with little finger;

- Thumb with index fin-

- Index finger with middle finger. ger;

\subsection{Palm orientation}

As in the case of the handshape, the palm orientation is specified for the dominant hand in the one-hand sign, for both hands in the two-hand signs. Then the ontology has a relation between One-hand sign and Palm orientation for the dominant hand, and two relations between Two-hand sign and Palm orientation, one for the dominant hand and the other for the nondominant hand.

According to the paper dictionary, the palm orientation has to be specified for the initial position of the hand/hands. To express this, the ontology has a relation between the Palm orientation class and the Hand or hands initial position class.

Then the Palm orientation class is subdivided into:

- Palm towards the - Palm away from the signer; signer;

- Palm down;

- Palm left;

- Palm up;

- Palm right.

\subsection{Hands relational position}

The relational position of the hands pertains to twohand signs (see Table 11; thus the ontology has a relation between the Hands relational position and Two-hand sign classes. The Hands relational position class is subdivided into into three main classes: Right-left contact, e.g., contact with elbow; Right-left distance, e.g., hands interlinked; Right-left spatial position, e.g., one hand inside the other. These are further partitioned and related as specified in (E-LIS ontology, 2006).

\section{CONCLUDING REMARKS}

Regarding the handshape, location and movement formational components of signs, some observations are in order. They are not part of the ontology in its current form. In the future, after collecting more detailed information of the LIS domain, the observa- tions could be turned into axioms of the ontology e.g., in the form of description logic formulae; they could be used to improve and shorten up the sign composition process. We discuss some of them as follows.

\subsection{Handshape and location}

A relevant observation (Volterra, 2004) is that, in the asymmetric signs, the open-hand and closed-fist handshapes are very frequent for the non-dominant hand. Alas, as such this piece of information is still too vague for us; first, the exceptions to the rule need to be pin down; then the rule, modulo the exceptions, can be added to the ontology as an axiom. In this manner the user who is looking up for an asymmetric sign can only select those handshapes for the nondominant hand, modulo the exceptions.

The dominant hand and the non-dominant hand may have the same location. This is generally true for the simple two-hand signs. However, an exception to the above rule is given by signs with nondominant hand, arm or wrist as locations - see 108.1 in (Radutzky, 2001). As for the composed signs, we found some counter-examples where the locations for the dominant hand and non-dominant hand are different (see 197.3 and 184.2 in (Radutzky, 2001)).

\subsection{Movement}

As stated in Table 1, some components of the movement can concern the dominant hand while others concern the non-dominant hand (see 184.2 in (Radutzky, 2001)), but 'which is which' is unclear. However, if the movement is composed of relational movement components (e.g., hands away from each other), the movements of the hands are clearly specified and this information can be turned into axioms.

By careful inspection of (Radutzky, 2001), one can see that most of the two-hand signs with identical handshape and palm orientation have symmetric or alternating movements (e.g., see 741.1 or 140.3 in (Radutzky, 2001)). Currently, this cannot be specified as a general rule; by way of contrast, see 630.2 in (Radutzky, 2001).

\section{THE ONTOLOGY-DRIVEN DICTIONARY}

As claimed above, an ontology-driven dictionary allow us to develop a intensional navigation of the LISto-Italian dictionary, so that also non-experts can use the dictionary. But how? 
The first step of the development consisted in the creation of the domain ontology explained above; this analyses, makes explicit to all and represents how signs are decomposed in an unambiguous way. By revealing implicit information or wrong assumptions, the domain ontology helped improve the flow of information within the e-LIS team. As such, it played an important role in the requirement analysis and conceptual modelling phase of the e-LIS database schema. The ontology was developed in ICOM hence we could use a DIG-enabled DL reasoner to check that the decomposition rules of the ontology are consistent (Fillottrani et al., 2006).

Moreover, the domain ontology serves as the basis for the definition of the application ontology which is tuned to the data present in the e-LIS database. The application ontology then becomes the input of a DIG-enabled query tool like (Catarci, T. et al., 2004); the two main modules of this tool are the Compose module for assisting the user in effectively composing a query, and the Query module for directly specifying the data which should be retrieved from the data sources. In particular, with input the LIS ontology, Compose will propose sign components (ontology classes) which are related to the user's current selection, as specified in the ontology; e.g., if the user selects "one-hand sign" then the query tool will not show "hands relational position" as next possible choice to the user, because the ontology does not relate these concepts. We refer the reader to (Catarci, T. et al., 2004) for more on the query tool and its intergration with a database.

The visualisation tool is the other main component of the ontology-driven dictionary. The visualisation of the composition, query process and results should meet the needs of the different users of the dictionary; for instance, deaf users are 'visual reasoners' (Sacks, 1989) hence their visual reasoning strategies must be considered as well.

\section{RELATED WORK}

Electronic dictionaries for SLs offer numerous advantages over conventional paper dictionaries; they can make use of the multimedia technology, e.g., video can be employed for rendering the hand movements. In the remainder, we review available electronic dictionaries from an SL to the verbal language of the country of origin, which are of interest to our work.

The bilingual Multi-Media Dictionary for American SL (MM-DASL) (Wilcox, 2003) developed a special user interface, with film-strips or pull-down menus. This allows users to look up for a sign only reasoning in terms of its visual formational components, that is, the Stokoe ones (handshape, location and movement); search for signs is constrained via linguistic information on the formational components. Users are not required to specify all the sign's formational components, nevertheless there is a specific order in which they should construct the query. Since the domain ontology embodies semantic information on the classes and relations of sign components for the e-LIS dictionary, the ontology can be used as the basis for an ontology-driven dictionary which forbids constraint violations - see Section 5 Platform independence of the system was a problem for MM-DALS; this is an issue the e-LIS team is taking into account, thus the choice of having the e-LIS dictionary as a web application. The profile of the expected user was never analyzed, whereas eLIS aims at a dictionary non-experts of LIS can use, as explained in Section 1. Last but not least, the MM-DALS team experienced communication problems among linguists and programmers; the domain ontology described in this paper has been helpful in this respect, contributing to make explicit relevant information and correcting assumptions about the sign decomposition rules for the e-LIS dictionary.

A bidirectional dictionary for Flemish SL, still being elaborated, is (Flemish Dictionary, 1999). Users are presented with images of the body parts involved in the sign formation; by clicking on a body part, the user is presented the list of all symbols available for that part. However, non-experts of the adopted representation system, namely SignWriting, cannot easily use this dictionary. Non-experts are not guided through the composition process, thus it is easy for them to choose combinations of sign components leading to meaningless gestures, that is, not corresponding to any Flemish SL sign. Similar remarks apply to other on-going transcription-based dictionaries, e.g., see (Vettori, 2006); they are mainly suited to experts of SL and the adopted transcription system.

To the best of our knowledge, ours is the first ontology developed for a sign language dictionary so far.

\section{CONCLUSIONS}

In the initial phase of the e-LIS project, the web dictionary from LIS to verbal Italian was intended for expert signers, only. This restriction is no longer valid; the users of the web dictionary of e-LIS can also be non-experts of LIS, willing to learn it. These users do not know how to compose sign components; as made clear in Section 2 it is not realistic to expect that these users will master the transcription system for decom- 
posing and retrieving signs from the web dictionary.

The domain ontology presented in this paper analyses and represents the rules for the decomposition of signs of LIS as explained in Sections 3 and 4 it was developed in a top-down manner starting from (Radutzky, 2001), under the guidance of linguists and deaf users of e-LIS (see Section 2).

The domain ontology already brought the following benefits to the e-LIS project (see Section 5): it made explicit domain assumptions and facilitated knowledge sharing in the e-LIS team; it helped in the requirement analysis and conceptual modelling of the e-LIS database schema; the decomposition rules behind the ontology were checked to be consistent. Moreover, an application ontology, tuned to the data present in the e-LIS database, is being built on top of the domain ontology presented here; a query tool like (Catarci, T. et al., 2004) can then be employed to assist the users in their sign composition. We are also working on the visualisation of the composition, query process and results.

The next milestone of the project will be an evaluation of the ontology-driven dictionary with real users to assess the usability of the dictionary. More linguistic knowledge of the LIS domain should also be gathered so as to enrich the ontology hence better assist users in their sign composition (see Section 4), and to devise good ranking criteria of the visualised results.

As remarked in Section 2, the analysis of the representations of SLs in written form is a topic of current research per se. At present, this goes beyond the scope of our work, which is mainly of experimental nature. After this experimental phase, we can turn to more foundational work with a deeper analysis of the written representations of SLs.

\section{ACKNOWLEDGEMENTS}

We thank for their contributions: J. Anderson; P. Dongilli; P. Fillottrani; E. Franconi; S. Tessaris; M. Tomkowicz; C. Vettori; C. Zanoni.

\section{REFERENCES}

Catarci, T., Dongilli, P., Di Mascio, T., Franconi, E., Santucci, G., and Tessaris, S. (2004). An Ontology Based Visual Tool for Query Formulation Support. In Proceedings of the 16th Biennial European Conference on Artificial Intelligence, ECAI 2004, Valencia, Spain.

E-LIS ontology (2006). URL: http://elis.eurac.edu/ WebSites/ELIS/Doc/eLISontology.pdf Last visit: Nov. 2006

E-LIS project (2004). URL: http://elis.eurac.edu/ Last visit: Nov. 2006.
Fillottrani, P., Franconi, E., and Tessaris, S. (2006). The New ICOM Ontology Editor. In International Workshop on Description Logics, DL 2006.

Flemish Dictionary (1999). URL: http://gebaren. ugent.be/visueelzoeken.php? Last visit: Nov. 2006

Garcia, B. (2006). The methodological, linguistic and semiological bases for the elaboration of a written form of French Sign Language (LSF). In Proceedings of the Workshop on the Representation and Processing of Sign Languages, LREC 2006.

Guarino, N. (1998). Formal Ontology in Information Systems. In Formal Ontology in Information Systems, FOIS 1998

Pizzuto, E., Rossini, P., and Russo, T. (2006). Representing signed languages in written form: questions that need to be posed. In Proceedings of the Workshop on the Representation and Processing of Sign Languages, LREC 2006.

Radutzky, E. (2001). Dizionario bilingue elementare della lingua italiana dei segni. Kappa.

Sacks, O. (1989). Seeing Voices: a Journey into the World of the Deaf. Vintage Books. (Italian version: "Vedere Voci”, Adelphi, 1999).

Stoke, W., Casterline, D., and Croneberg, C. (1965). A dictionary of American sign language on linguistic principles. Linstok Press.

Vettori, C., editor (2006). Representation and Processing of Sign Languages. Lexicographic Matters and Didactic Scenarios. Fifth International Conference on Language Resources and Evaluation. Post-conference workshop. Paris: ELRA.

Vettori, C., Streiter, O., and Knapp, J. (2004). From Computer Assisted Language Learning (CALL) to sign language processing: the design of e-LIS, an electronic bilingual dictionary of Italian sign language and Italian. In Proceedings of the Workshop on the Representation and Processing of Sign Languages, LREC 2004.

Volterra, V., editor (2004). La lingua dei segni italiana. La comunicazione visivo-gestuale dei sordi. Il Mulino.

Wilcox, S. (2003). The multimedia dictionary of American sign language: Learning lessons about language, technology, and business. Sign Language Studies, 3(4):379-392. 